\title{
Über die Temperaturabhängigkeit der inneren Dämpfung von weichgemachtem Polymethacrylsäuremethylester
}

\author{
Von E. Jenckel und K.-H. Illers
}

Aus dem Institut für theoretische Hüttenkunde und physikalische Chemie der Techn. Hochschule Aachen

(Z. Naturforschg. 9a, 440-450 [1954]; eingegangen am 2. Februar 1954)

\begin{abstract}
Es wurde die innere Dämpfung von mit Butylacetat und Chloroform weich gemachtem Polymethacrylsäuremethylester im Temperaturbereich von -60 bis $+90^{\circ}$ mit einem Torsionspendel bei der konstanten Frequenz von $0,5 \mathrm{~Hz}$ untersucht. Das logarithmische Dekrement weist beim reinen Polymerisat ein sehr hohes Maximum bei etwa $100^{\circ}$ und ein kleines Nebenmaximum bei etwa $15^{\circ}$ auf. Bei Weichmacherzusatz verschieben sich beide Maxima zu tieferen Temperaturen, jedoch das größere in stärkerem Ausmaß, so daß es das kleinere schließlich überholt. In der Nähe der Einfriertemperatur treten nach geringerer Abkühlungsgeschwindigkeit oder nach Tempern Zeiteffekte auf, derart, daß das Minimum zwischen den beiden Maxima tiefer zu liegen kommt. Die Versuche lassen sich mit Hilfe eines zweifachen Maxwellschen Modells auswerten. Die Relaxationszeiten ändern sich nach der zu erwartenden Formel $\tau=\tau_{0} \exp (A / R T)$, jedoch ist die Aktivierungsenergie $A$ im Glaszustand fast 10-mal kleiner als in der Gleichgewichtsschmelze. Das Maximum des Dekrements ist durch das Verhältnis von Relaxationszeit zu Frequenz bedingt, die Einfriertemperatur dagegen durch die Geschwindigkeit der vorherigen Abkühlung. Durch Zusatz von Weichmacher nimmt die Aktivierungsenergie der Schmelze stark $\mathrm{ab}$ und ebenfalls $\tau_{20}$, entsprechend der für alle Präparate gültigen einfachen Beziehung $\log \left(1 / \tau_{20}\right)=0,57 A_{2}+2$.
\end{abstract}

$\mathrm{B}$ ei der Untersuchung des plastisch-elastischen Verhaltens von hochmolekularen Stoffen haben sich in den vergangenen Jahren mehr und mehr die dynamischen Versuchsmethoden in den Vordergrund geschoben. Zahlreiche Apparaturen sind entwickelt worden, in denen entweder ein Probekörper zu erzwungenen Schwingungen bei vorgegebener periodischer Kraft oder Verformung erregt wird, oder indem man ihn zu einer freien Schwingung anregt und deren Abklingen infolge innerer Dämpfung beobachtet $^{1-4}$. Insbesondere zeichnet sich die Methode der freien Torsionsschwingungen ${ }^{5-7}$ durch große Meßgenauigkeit, einfache Meßmethodik und geringen apparativen Aufwand aus. Sowohl in rein wissenschaftlicher als auch in anwendungstechnischer Hinsicht interessiert sowohl die Temperaturabhängigkeit als auch die Frequenzabhängigkeit der Kenngrößen, die das plastisch-elastische Verhalten des untersuchten Stoffes beschreiben, nämlich des dynamischen Elastizitätsmoduls $E$ als Maß der Stoffelastizität und der Verformbarkeit oder des log-Dekrementes $\Lambda$ als Maß der Energiedissipa-

${ }^{1}$ R. S. Marvin, Industr. Engng. Chem. 44, 696 [1952]. Dort auch ausführliche Literaturangaben.

2 J. D. Ferry u. E. R. Fitzgerald, J. Coll. Sci. 8, I [1953].

${ }^{3}$ L. E. Nielsen, Rev. Sci. Instrum. 22, 690 [1951].

${ }^{4}$ W. Lethersich, J. Sci. Instrum. 27, 303 [1950]. tion, d. h. des Verlustes, der bei Beanspruchung im Material auftritt. Um ein vollständiges Bild des dynamischen Verhaltens des Kunststoffes zu bekommen, ist es notwendig, die Temperatur und insbesondere die Frequenz in weiten Grenzen zu variieren. Die Dimensionen der Versuchskörper und die Größe der mitschwingenden Massen erlaubt bei den freien Torsionsschwingungen jedoch nur eine Variation der Versuchszeiten um max. etwa 3 Zehnerpotenzen. Die hier mitgeteilten Versuche beschränken sich deshalb bewußt auf eine etwa konstante Frequenz von $0,5 \mathrm{~Hz}$. Es wird daher die Abhängigkeit des plastisch-elastischen Verhaltens von der Frequenz sowohl in den Versuchen als auch in der Auswertung ausdrücklich offen gelassen.

\section{Versuchsergebnisse}

a) Die Dämpfung in Abhängigkeit von der Temperatur

In dieser Arbeit wurde an Polymethacrylsäuremethylester (PMA) und verschiedenen Lösungen

5 E. Jenckel, H. Hertog u. E. Klein, Z. Naturforschg. 8 a, 255 [1953].

${ }^{6} \mathrm{~K}$. Wolf, Kunststoffe 41, 2 [1951].

${ }^{7}$ W. K uhn u. O. K ünzle, Helv. Chim. Acta 30, 839 [1947]. 
bis zu 20\% Lösungsmittel (Weichmacher) die innere Dämpfung bei freien Torsionsschwingungen etwa konstanter Frequenz untersucht. Als Maß derselben wurde das log. Dekrement $\Lambda$ benutzt (siehe IV.).

Beim reinen PMA weist $\Lambda$ abhängig von der Temperatur zwei Maxima auf (Abb. 1). Bei einer Temperatur von ca. $100^{\circ} \mathrm{C}$ tritt ein großes (Haupt-) Dämpfungsmaximum auf, wie es auch bei vielen anderen Stoffen gefunden wurde ${ }^{3,5,6,8}$. Dort wird die Dämpfung unmeßbar groß. Der Hauptaugenmerk wurde auf ein zweites, wesentlich kleineres Dispersionsgebiet gelegt, welches bei $15^{\circ} \mathrm{C}$ ein Dämpfungsmaximum mit $\Lambda=0,33$ besitzt. Zwischen beiden Maxima wird bei $50^{\circ} \mathrm{C}$ ein Minimum mit $\Lambda=0,25$ durchlaufen. Die Existenz dieses sekundären Dispersionsgebietes wurde bereits von Schmieder und Wolf ${ }^{9}$, Iwayanagi und Hideshima ${ }^{10}$ sowie Heyboer, Dokking und Staver mann ${ }^{22}$ mitgeteilt, doch liegen genauere Untersuchungen und Auswertungen noch nicht vor. Deswegen wurde bei allen anderen Messungen der Temperaturbereich auf Temperaturen $<100^{\circ} \mathrm{C}$ beschränkt und der abfallende Ast oberhalb der Temperatur des Hauptmaximums nicht erfaßt.

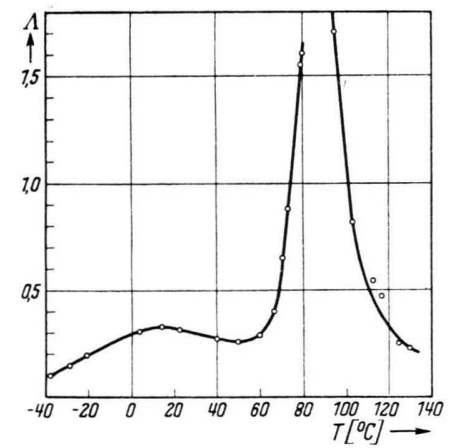

Abb. 1. Das log. Dekrement $\Lambda$ im System PMA $+3 \%$ Toluol.

Weiter wurde die Dämpfung gleichartiger Probekörper gemessen, die mit Toluol, Aceton, Butylacetat und Chloroform in verschiedenen Konzentrationen weich gemacht waren. Die Ergebnisse sind in den Abb. 2, 3, 4 dargestellt (Abkühlungsgeschwindigkeit $\left.0,5^{0} / \mathrm{min}\right)$. Bei Weichmacherzusatz rückt die gesamte Dämpfungskurve zu tieferen Temperaturen. Bis zu Konzentrationen von ca. 15 Gew.- \% verschiebt sich der aufsteigende Ast des

${ }^{8}$ A. W. Nolle, J. Polymer. Sci. 5, 1 [1950].

${ }^{9}$ K. Schmieder u. K. Wolf, Kolloid-Z. 127, 62 [1952].
Hauptmaximums einfach parallel. Bei weiterem $\mathrm{Zu}$ satz von Weichmacher werden die Kurven flacher und die Dämpfung im Hauptmaximum wird wahrscheinlich kleiner. Das sekundäre Dämpfungsmaximum verschiebt sich ebenfalls zu tieferen Temperaturen, aber nicht so rasch wie das Hauptmaximum, so daß es von diesem eingeholt und schließlich überdeckt wird. Bei Konzentrationen> ca. 20\% Weichmachergehalt ist es nur noch als kleine Ausbuchtung im aufsteigenden Ast des Hauptmaximums

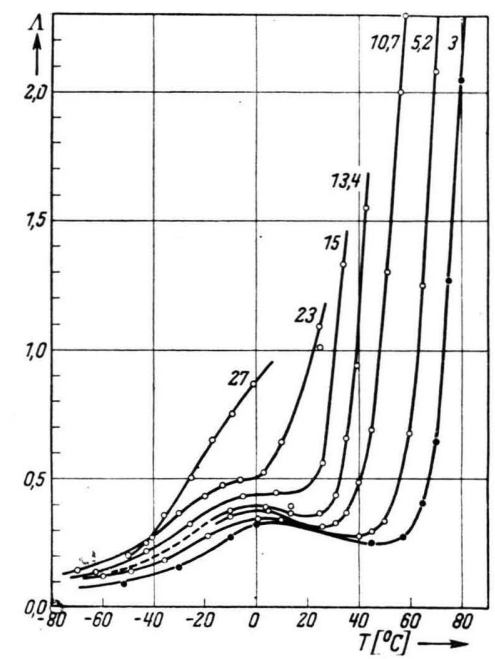

Abb. 2. Das log. Dekrement $\Lambda$ im System PMA - Butylacetat. Parameter: Gew.-\% Weichmacherzusatz.

zu erkennen. Von den verwendeten Weichmachern bewirkt Butylacetat die größte, Chloroform die kleinste Verschiebung der Dämpfungskurven zu tieferen Temperaturen (bezogen auf gleichen Zusatz in Gew.- \%), und zwischen beiden liegen Toluol und Aceton.

Eine Abhängigkeit vom Polymerisationsgrad (für $P=6000,3000$ und 1000) wurde nicht beobachtet. (Siehe Abschn. IV.)

Die Dämpfungskurven waren sehr gut reproduzierbar, wenn man von hohen zu tiefen Temperaturen maß und dabei eine konstante Abkühlungsgeschwindigkeit einhielt.

ß) Zeitliche Änderungen in der Umgebung der Einfriertemperatur

In einem Temperaturbereich zwischen $30^{\circ} \mathrm{C}$ und $60^{\circ} \mathrm{C}$ beobachtet man Zeiteffekte. Mißt man die

10 S. Iwayanagi u. T. Hideshima, J. Phys. Soc. Japan 8, 365 [1953]. 
Dämpfung von hohen zu tiefen Temperaturen fortschreitend, so erhält man in diesem Bereich bei jeder Temperatur Dämpfungswerte, die um so kleiner sind, je geringer die Abkühlungsgeschwindigkeit ist. Kühlt man also die Probe von Meßpunkt zu Meßpunkt langsam ab (ca. $0,1^{0} / \mathrm{min}$ ), so erhält

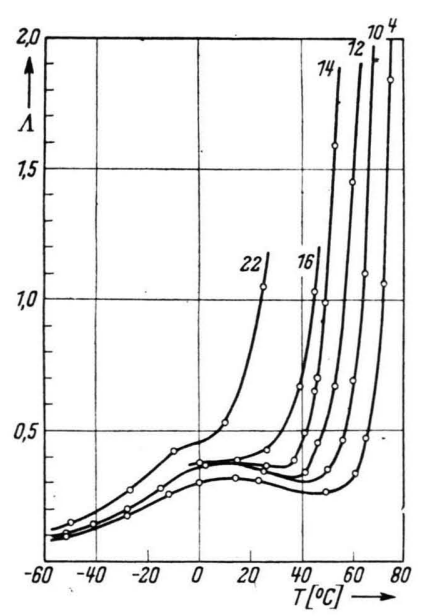

Abb. 3. Das log. Dekrement $\Lambda$ im System PMA-Chloroform. Parameter: Gew.-\% Weichmacherzusatz.

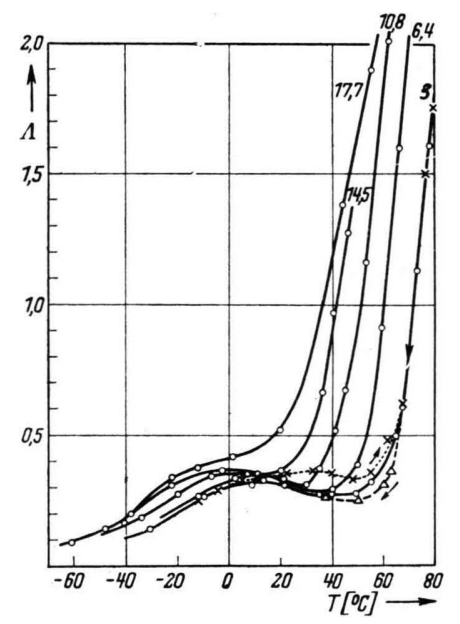

Abb. 4. Das log. Dekrement $\Lambda$ im System PMA-Toluol. Parameter: Gew.-\% Weichmacherzusatz. - - - geringere Abkühlgeschwindigkeit.

man z. B. eine Dämpfungskurve, wie sie in Abb. 4 gestrichelt gezeichnet ist $(0 \%$ Toluol). Sie verläuft unterhalb der Kurve, die mit größerer Abkühlungsgeschwindigkeit erhalten wurde $\left(0,5^{0} / \mathrm{min}\right.$; ausgezogene Kurve). Zur näheren Untersuchung der Zeiteffekte wurden Proben von einer Temperatur von $100^{\circ} \mathrm{C}$ ausgehend rasch auf verschiedene Temperaturen $\left(59^{\circ} \mathrm{C}, 63^{\circ} \mathrm{C}, 71^{\circ} \mathrm{C}\right)$ abgekühlt und bei diesen
Temperaturen die Abnahme der Dämpfung mit der Zeit beobachtet. Das Abklingen mit der Verweilzeit $t$ kann man in die empirische Gleichung (Abb. 5)

$$
\Lambda=\Lambda_{\infty}+B e^{-t / \tau^{*}}
$$

kleiden, worin $\Lambda_{\infty}$ der schließlich erreichte Grenzwert und $\tau^{*}$ die Zeitkonstante des Abklingens bedeutet. $\left(\Lambda_{\infty}+B\right)$ ist die sich spontan bei Temperaturänderung einstellende Dämpfung (Verweilzeit $t=0)$.Werte für $B$ können aus diesen Experimenten nicht entnommen werden, da man wegen der Trägheit der Temperatureinstellung in der Probe nicht den Nullpunkt der Verweilzeit kennt.

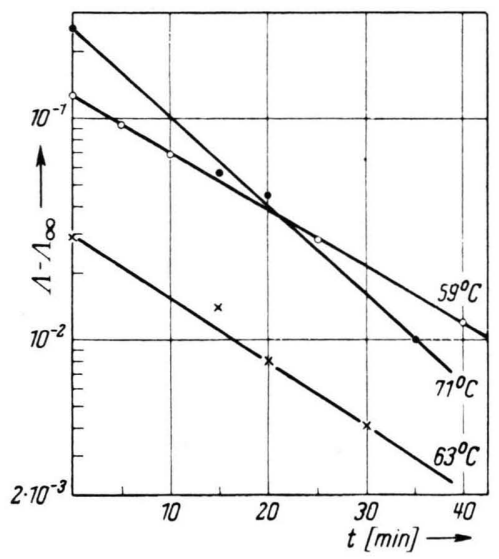

Abb. 5. Das Abklingen von $\Lambda$ mit der Verweilzeit $t$.

Bei tiefen Temperaturen $\left(<50^{\circ} \mathrm{C}\right)$ ist $\tau^{*}$ so groß, daß man das Abklingen der Dämpfung in zugänglichen Zeiten nicht mehr verfolgen kann. Nur unmittelbar unterhalb der Einfriertemperatur lassen sich Beobachtungen machen*.

Die Temperaturabhängigkeit von $\tau^{*}$ kann man darstellen als :

$$
\tau^{*}=\tau_{0}^{*} e^{A_{\mathrm{N}} / R T} .
$$

Zahlenangaben in Tab. 1 .

\begin{tabular}{|c|c|c|c|}
\hline & $59^{0} \mathrm{C}$ & $63^{0} \mathrm{C}$ & $71^{0} \mathrm{C}$ \\
\hline$\Lambda_{\infty}$ & 0,322 & 0,353 & 0,55 \\
$\tau^{*}$ & $16,8 \mathrm{~min}$ & $14,5 \mathrm{~min}$ & $10,8 \mathrm{~min}$ \\
\hline \multicolumn{3}{|c|}{$\tau_{0}^{*}=3,2 \cdot 10^{-4} \mathrm{~min} ; A_{\mathrm{N}}=8,8 \mathrm{kcal} / \mathrm{Mol}$} \\
\hline
\end{tabular}

Tab. 1. Temperaturabhängigkeit von $\Lambda_{\infty}$ und $\tau^{*}$.

* Mißt man dagegen von tiefen zu hohen Temperaturen, so erhält man bei gleichem Temperaturanstieg von $0,5^{0} / \mathrm{min}$ im allgemeinen Dämpfungskurven, die über den mit fallender Temperatur gemessenen liegen (Abb. 4, punktiert), vgl. hierzu auch G. Rehage und E. Jenckel, Über Hysterese-Effekte eben unterhalb der Einfriertemperatur, noch unveröffentlicht. 
$\gamma)$ Der dynamische Torsionsmodul in Abhängigkeit von der Temperatur

Der Torsionsmodul $G$ ist definiert als Proportionalitätsfaktor zwischen Spannung und Verformung beim Scherversuch:

$$
s=G \cdot \gamma
$$

( $s=$ Schubspannung, $\quad \gamma=$ Schubverformung). Bestimmt man $G$ aus einem statischen Torsionsversuch, so tritt an die Stelle von $s$ das Drehmoment $M$.

$$
M=G \frac{r^{4} \pi}{2} \frac{\alpha}{l}
$$

$(r=$ Radius der zylindrischen Probe; $l=$ Länge, $\alpha=$ Verdrehung des freien Endes im Bogenmaß). Für rechteckige Proben ist eine Querschnittsverwölbung in Rechnung zu stellen, und man erhält nach De Saint-Venant ${ }^{11}$ :

$$
M=G \frac{b d^{3}\left(1-0,63 \frac{b}{d}\right)}{3} \frac{\alpha}{l} \text { für } \frac{d}{b}<\frac{1}{3} .
$$

Bestimmt man den Torsionsmodul nach einem dynamischen Meßverfahren, so wird wegen

$$
M / \alpha=D \text { (Direktionsmoment) }
$$

und

$$
D=4 \pi^{2} \Theta / \vartheta^{2}
$$

$(\vartheta=$ Schwingungsdauer, $\Theta=$ Trägheitsmoment der mitschwingenden Massen)

aus (4)

$$
G=\frac{8 \pi l \Theta}{\vartheta^{2} r^{4}},
$$

bzw. aus (5) für rechteckige Querschnitte

$$
G=\frac{12 \pi^{2} l \Theta}{\vartheta^{2} b d^{3}\left(1-0,63 \frac{d}{b}\right)}=\frac{3 l}{b d^{2}\left(1-0,63 \frac{d}{b}\right)} D .
$$

Nach den Gln. (3) - (7) sollte der statische Modul gleich dem dynamischen sein. Diese Übereinstimmung gilt jedoch nur für den rein elastischen Stoff. Sobald Verluste auftreten, ändert sich der $G$-Modul mit der Versuchszeit. Für den statischen $G$-Modul ist die Zeit maßgebend, die zwischen Aufbringen der Spannung und Messung der Verformung verstrichen ist, während der dynamische Modul von der Frequenz abhängig wird. Grob gesprochen ist nur dann $G_{\text {stat }}=G_{\text {dyn }}$, falls die Versuchszeiten im statischen und dynamischen Versuch die gleichen sind, und für die Bestimmung des auf rein elastischer (Hookescher) Verformung beruhenden G-Moduls ist es notwendig, in beiden Fällen die Versuchs-

${ }^{11}$ Vgl. Kohlrausch, Praktische Physik, 19. Aufl., Teubner 1950, Bd. 1, S. 84. zeit sehr viel kleiner als die Relaxationszeit zu wählen.

Dieser Abhängigkeit des Moduls von der Versuchszeit wird besonders in der ausländischen Literatur durch Einführung des komplexen dynamischen Moduls Rechnung getragen:

Darin ist

$$
G^{*}(\omega)=G^{\prime}(\omega)+i G^{\prime \prime}(\omega) .
$$

$$
\begin{aligned}
& G^{\prime}=\frac{\text { Komponente der Spannung, die mit der Verformung in Phase ist }}{\text { Verformung }}, \\
& G^{\prime \prime}=\frac{\begin{array}{c}
\text { Komponente der Spannung, die mit der Verformungsgeschwin- } \\
\text { digkeit in Phase ist }
\end{array}}{\text { Verformungsgeschwindigkeit }} .
\end{aligned}
$$

Dieser komplexe dynamische Modul ist nur für sinoder cos-förmige Spannung und Verformung definiert.

Der absolute Betrag des dynamischen Moduls

$$
\left|G^{*}\right|=\sqrt{G^{\prime 2}+G^{\prime 2}}
$$

ist das Verhältnis der Gipfelwerte von Spannung und Verformung.

Der nach den Gln. (3) - (7) bestimmte G-Modul stellt aber ebenfalls nichts anderes dar als das Verhältnis von max. Spannung und max. Verformung. Will man also Ergebnisse vergleichen, die im komplexen dynamischen Modul ausgedrückt sind mit solchen, die nach den Gln. (3)-(7) berechnet wurden, so muß man auf Gl. (9) zurückgreifen.

Ferner gilt für den Phasenwinkel $\varphi$ zwischen Spannung und Verformung

$$
\operatorname{tg} \varphi=G^{\prime \prime} / G^{\prime}
$$

und für den Zusammenhang mit dem log. Dekrement

$$
\Lambda=\pi \operatorname{tg} \varphi=\pi G^{\prime \prime} / G^{\prime} \text { für } \Lambda \leqq 0,6 .
$$

Errechnet man nach Gl. (7) aus den gemessenen Schwingungsdauern den Torsionsmodul, so ergibt sich eine Temperaturabhängigkeit des Torsionsmoduls, wie sie in Abb. 6 für das System PMAAceton für einige Weichmacherkonzentrationen dargestellt ist. Diese Darstellung ist im Prinzip vollkommen gleichwertig den Dämpfungskurven. Auch das sekundäre Dispensionsgebiet macht sich hier bemerkbar. Nach ${ }^{12}$ gibt diese Darstellung aber nicht den Torsionsmodul von PMA schlechthin wieder, sondern nach oben Gesagtem den dynamischen Modul nach Gl. (9), der, je nachdem bei welcher Tempe-

12 E. Klein u. E. Jenckel, Z. Naturforschg. 7a, 801 [1952]. 
ratur gemessen, von mehr oder weniger großen Relaxationseinflüssen überdeckt ist.

Im Prinzip kann auch die Darstellung des Torsionsmoduls nach Abb. 6 zur Auswertung herangezogen werden, doch ist eine theoretische Auswertung an Hand der Dämpfungskurven vorzuziehen, da diese mit größerer Genauigkeit zu bestimmen sind.

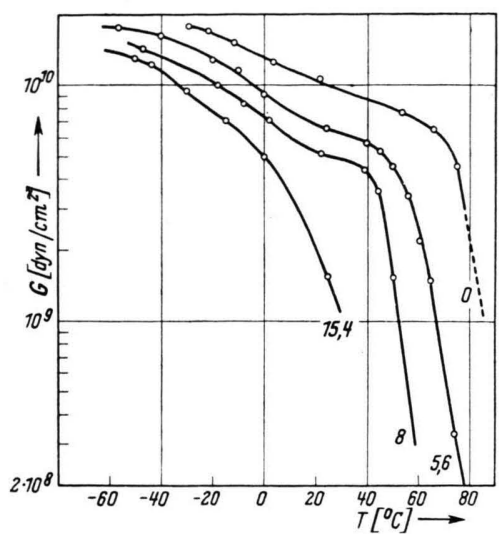

Abb. 6. Temperaturabhängigkeit des Torsionsmoduls $G$ im System PMA-Aceton. Parameter: Gew.-\% Weichmacherzusatz.

\section{Auswertung mit Hilfe Maxwellscher} Modelle

\section{a) Modelltypen}

Wie bei vielen anderen Problemen ist es auch hier nützlich, die experimentellen Befunde in ein Modell einzukleiden und an Hand eines solchen Modells das Verhalten des Stoffes zu studieren. Als Grundeinheit eines Modells wurde das sog. einfache Maxwellsche Modell ${ }^{13}$ und verschiedene Kombinationen desselben benutzt (Abb. 7).

Das Direktionsmoment der Feder $D$ ist das Sinnbild der reversiblen elastischen Komponente, die Relaxationszeit $\tau$ das der irreversiblen Komponente. Jedes (hintereinandergeschaltete) Wertepaar $D_{i}, \tau_{i}$ soll zu einer anderen Art von ,Bindungsmechanismus" gehören. Wendet man diese Modelle auf freie Torsionsschwingungen an, indem man die Bewegungs-Differentialgleichungen aufstellt, so kann man aus deren Lösungen das log. Dekrement $\Lambda$ als Funktion von $D_{i}, \tau_{i}$ ausrechnen. Dies ist in einer früheren Arbeit ${ }^{14}$ geschehen, auf die hier ver-

${ }^{13}$ E. Jenckel, Kolloid-Z. 134, 47 [1953].

14 E. Klein u. E. J enckel, Z. Naturforschg. 7a, 305 [1952].

* Die mit einem Stern versehenen Gln. beziehen wiesen sein soll. Für die einzelnen Modelle ergibt sich dabei folgendes Bild:

Ein plast.-elast. Mechanismus (einfaches Maxwellsches Modell) (Abb. 7 a)

$$
\Lambda=f\left(\tau, \vartheta_{0}\right) \text {. }
$$

Ein plast.-elast. und ein rein elastischer Mechanismus (1 1 $1 / 2$-faches Maxwellsches Modell) (Abb. 7 b)

$$
\Lambda=f\left(\tau, D_{1} / D_{2}, \vartheta_{0}\right) \text {. }
$$

Zwei plast.-elast. Mechanismen (zweifaches Maxwellsches Modell) (Abb. 7 c)

$$
\Lambda=f\left(\tau_{1}, \tau_{2}, D_{1} / D_{2}, \vartheta_{0}\right) \text {. }
$$

Bei der Einführung dieser Modelle wird vorausgesetzt, da $\beta$ die Federkonstante $D$ als $\mathrm{Ma} \beta$ der reversibel aufgespeicherten Kraft nicht von der Temperatur abhängt, abgesehen von geringfügiger Änderung des statistischen $G$-Moduls proportional mit $T$,
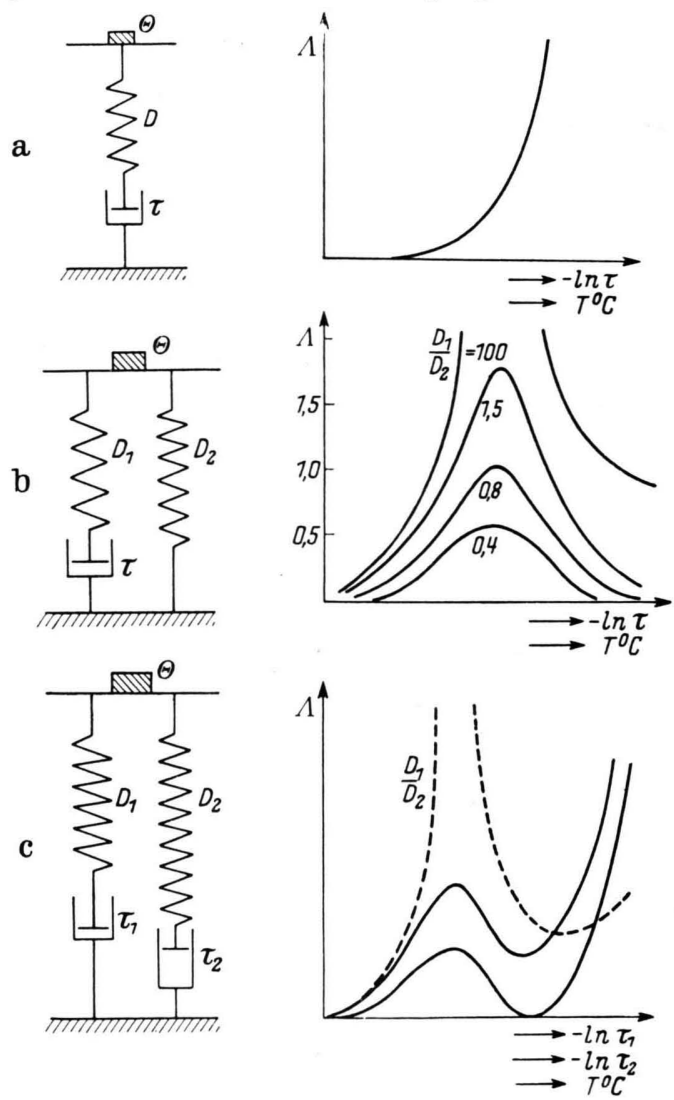

Abb. 7a-c. Verschiedene Maxwellsche Modelle mit den zugehörigen berechneten Dämpfungskurven.

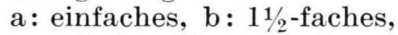

c: 2-faches Maxwellsches Modell.

sich auf die entsprechenden, ausführlichen Gln. in ${ }^{14}$, die hier nur schematisch wiedergegeben sein mögen. Dort ist statt der Masse $m$ das Trägheitsmoment $\Theta$ einzusetzen. 
und die Temperaturabhängigkeit des Modells lediglich durch die Temperaturabhängigkeit der Viskosität der , dashpots" zustande kommt. Da nach Maxwell gilt

$\begin{aligned} G \tau & =\eta \\ \text { und da } \quad \eta & =\eta_{0} \cdot e^{A / R T}\end{aligned}$

muß bei Richtigkeit obiger Annahme gelten

$$
\tau=\tau_{0} \cdot e^{A / R T} \text { mit } \tau_{0}=\eta_{0} / G .
$$

Dieser G-Modul, der sich nach Gl. (7) aus dem Direktionsmoment (Federkonstante) $D$ des Modells berechnet, ist nun keineswegs der dynamische Modul, der ja von Frequenz und Temperatur abhängt, sondern dessen Grenzwert bei sehr tiefen Temperaturen $(T \rightarrow 0 ; \Lambda \rightarrow 0$; rein elastisches Verhalten). Es soll deshalb hier unter dem Modul derjenige verstanden werden, der aus der bei sehr tiefen Temperaturen gemessenen Schwingungsdauer $\vartheta_{0}$ nach Gl. (7) berechnet wird, d. h., die Relaxationseinflüsse machen sich nicht im Modul, sondern lediglich in der Dämpfung bemerkbar.

\section{$\beta$ ) Methode der Auswertung}

Die Anwendung des einfachen Maxwellschen Modells auf experimentelle Ergebnisse ist leicht. Die $\operatorname{des} 11 / 2$-fachen Modells wurde im wesentlichen bereits an anderer Stelle ${ }^{5}$ mitgeteilt. Danach wird zunächst der Gesamt-Modul aus $\vartheta_{0}$ und dann das Verhältnis $G_{1} / G_{2}=D_{1} / D_{2}$ aus der Höhe des Dämpfungsmaximums gemä $\beta$ der theoretischen Dämpfungsgl. $\left(9 \mathrm{c}^{*}\right)$ ermittelt. Das letztere Verfahren führt bereits zu umständlichen Rechnungen. Sie werden jetzt sehr einfach, nachdem durch numerische Auswertung dieser Gleichung gefunden wurde, daß für die Dämpfung im Maximum gilt:

$\Lambda_{\text {theor. max. }}=1,24 \frac{D_{1}}{D_{2}}+0,08$ für $0,1<\frac{D_{1}}{D_{2}}<2$.

Weiter wird durch Vergleich der experimentellen und theoretischen Dämpfungskurve jeder Temperatur eine Relaxationszeit $\tau$ zugeordnet. Auch hier führt eine einfache numerische Auswertung der Gl. (9 $\left.\mathrm{c}^{*}\right)$ zu der einfachen Beziehung

$$
\tau_{\Lambda_{\max }}=1,25 \vartheta_{0} \text { für } 10^{-3}<\vartheta_{0}<10^{1} \mathrm{sec} .
$$

Die beiden Gl. (11), (12) bieten eine große Vereinfachung bei der Auswertung experimenteller Dämpfungskurven insofern, als man jetzt nur noch die Kurvenschar nach Gl. (9 $\left.\mathrm{c}^{*}\right)$ auf der $\tau$-Achse parallel verschieben und diejenige Kurve auswählen muß, deren Höhe $\Lambda_{\max }$ [und also nach Gl. (11) auch $D_{1} / D_{2}$ ] mit dem experimentellen Wert übereinstimmt.

In dem hier untersuchten Beispiel muß man jedoch, wie der Vergleich von Abb. 1 und $7 \mathrm{c}$ lehrt, ein 2-faches Maxwellsches Modell zugrunde legen. Beim 2-fachen Modell erhält man $\Lambda_{\text {theor. }}$ auch nach Eliminierung von $\vartheta_{0}$ und $D_{1} / D_{2}$ als Funktion zweier Variabler, nämlich $\tau_{1}$ und $\tau_{2}$. Ein direkter Vergleich von experimenteller und theoretischer Kurve ist daher nicht möglich. Liegen jedoch die beiden Dispersionsgebiete weit genug auseinander, so könnte man sie getrennt beschreiben. Bei der verhältnismäßig tiefen Temperatur des sekundären Maximums wäre nämlich das zweifache Modell in ein solches mit $\tau_{2} \approx \infty$, d. h., in ein $11 / 2$-faches übergegangen, während andererseits bei der höheren Temperatur des zum Hauptmaximum aufsteigenden Astes wegen $\tau_{1} \rightarrow 0$ ein 1-faches Maxwellsches Modell mit $D_{2}$ und $\tau_{2}$ entstanden wäre. Man würde dann in der oben angedeuteten Weise die Werte $D_{1}, D_{2}$ und $\tau_{1}, \tau_{2}$ bestimmen können.

Im vorliegenden Beispiel des PMA sind jedoch die Dispersionsgebiete nicht hinreichend voneinander getrennt. Vielmehr sind in einem gewissen Temperaturbereich beim reinen PMA zwischen 20 und $60^{\circ}$ beide Mechanismen wirksam: $\tau_{1}$ ist noch nicht verschwindend klein gegenüber $\tau_{2}$ geworden. Die Dämpfung verschwindet dort nicht, die Dämpfungskurve durchläuft nur ein Minimum. Auch setzt sich, wie man leicht zeigen kann, die Dämpfung in diesem Zwischenbereich nicht additiv aus dem Anteil der einzelnen Mechanismen zusammen, so daß sich die Kurve nicht ,entzerren“ läßt.

Wir werden im folgenden die experimentellen Kurven derart auswerten, daß wir bei tiefen Temperaturen das 1 1/2-fache Modell mit $\tau_{1}, D_{1}, D_{2}$, bei hohen Temperaturen das einfache Modell mit $\tau_{2}, D_{2}$ zugrunde legen und zusehen, wie wir bei mittleren Temperaturen (Umgebung des Minimums) die experimentellen Werte mit dem zweifachen Modell $\left(\tau_{1}, \tau_{2}, D_{1}, D_{2}\right)$ wiedergeben können.

\section{$\gamma)$ Das reine Polymerisat}

Betrachtet man zunächst einmal eine Dämpfungskurve an reinem PMA (Abb. 8 ausgezogene Linie), so stellt sich durch Vergleich mit der theoretischen Dämpfungskurve gemäß Gl. (9a*) heraus, daß der aufsteigende Ast des Hauptmaximums in einem Temperaturbereich von ca. $20^{\circ}$ sich durch ein einfaches Maxwellsches Modell beschreiben läßt, d.h. im $\ln \tau-\mathbf{l} / T$-Diagramm ergibt sich eine Ge- 
rade (Abb. 9, links). Die Temperaturabhängigkeit von $\tau_{2}$ stellt sich dar als

$$
\tau_{2}=\tau_{20} e^{A_{2} / R T} .
$$

Ferner ergibt sich für das kleine Dämpfungsmaximum bis etwa herauf zu der Temperatur des Maximums im $\ln \tau_{1}-\mathbf{l} / T$-Diagramm eine zweite Gerade (Abb. 9, rechts), indem man nach Abb. $7 \mathrm{~b}$ und Gl. $\left(9 \mathrm{c}^{*}\right) \quad\left(1 \frac{1}{2}\right.$-faches Modell) diejenige theoretische Dämpfungskurve zugrunde legt, deren $\Lambda_{\max \text {. theor. }}$ $=\Lambda_{\text {max. exp. ist. }}$

Es gilt also wieder

$$
\tau_{1}=\tau_{10} e^{A_{1} / R T} .
$$

$\mathrm{Um}$ das Zwischengebiet $\left(10^{\circ} \mathrm{C}\right.$ bis $\left.60^{\circ} \mathrm{C}\right)$, in dem die beiden Mechanismen sich überlagern, durch ein Modell geschlossen darstellen können, muß man offenbar auf das zweifache Maxwellsche Modell zurückgreifen. Hier ist man gezwungen, $\tau_{1}$ und $\tau_{2}$ in Gl. $\left(9 b^{*}\right)$ einzusetzen und punktweise $\Lambda_{\text {theor. }}$ auszurechnen. Es liegt nahe, dazu diejenigen Wertepaare $\tau_{1}, \tau_{2}$ zu nehmen, die man durch geradlinige Extrapolation von $\tau_{1}$ zu höheren und $\tau_{2}$ zu tieferen Temperaturen im $\ln \tau-1 / T$-Diagramm (Abb. 9, punktiert), also gemäß Gl. (13) und (14) erhält. Das bedeutet, daß man sowohl für $\tau_{1}$ als auch für $\tau_{2}$ in dem Zwischenbereich gleiche Temperaturabhängigkeit voraussetzt wie unter- bzw. oberhalb. Führt man dies durch, so errechnet man eine Dämpfungskurve, die in dem fraglichen Zwischenbereich unterhalb der experimentellen Kurve verläuft (Abb. 8, punktiert). Übereinstimmung ist auch dann nicht zu erzielen, wenn man $\tau_{2}$ linear extrapoliert und für $\tau_{1}$ willkürliche Werte einsetzt. Wohl aber gelangt man zu einer befriedigenden Darstellung, wenn man $\tau_{1}$ linear extrapoliert und einen Wert $\tau_{2}$, den wir jetzt* $\tau_{2}{ }^{\prime}$ nennen wollen, frei wählt. Diejenigen $\tau_{2}{ }^{\prime}$-Werte, die $\Lambda_{\text {theor. }}=\Lambda_{\text {exp. }}$ machen, logarithmisch gegen die reziproke Temperatur aufgetragen, liegen exakt auf einer zweiten Geraden von wesentlich geringerem Anstieg (Abb. 9, gestrichelt), die die erste Gerade bei einer Temperatur von $62^{\circ} \mathrm{C}$ schneidet. Diesen Schnittpunkt sehen wir als die Einfriertemperatur (vgl. w. u.) an. Berücksichtigt man noch die in den Probekörpern enthaltenen Lösungsmittelreste (siehe Abschn. IV), so gelangt man zu einer Einfriertemperatur des wirklich reinen PMA von

* Der Index' bezieht sich im folgenden immer auf den eingefrorenen Glaszustand.

15 E. Jenckel, Kolloid Z. 120 [1951].

${ }^{16} \mathrm{~J}$. L. Melchior u. A. A. Petrauskas, Industr. Engng. Chem. 44, 4 [1952]. $73,5^{\circ} \mathrm{C}$. Dieser Wert steht in guter Übereinstimmung mit Werten, die aus mehreren anderen Methoden gewonnen wurden ${ }^{15-18}$.

Auf ähnliche Weise wurden unter Anwendung des $1 \frac{1}{2}$-fachen Maxwellschen Modells Dämpfungskur-

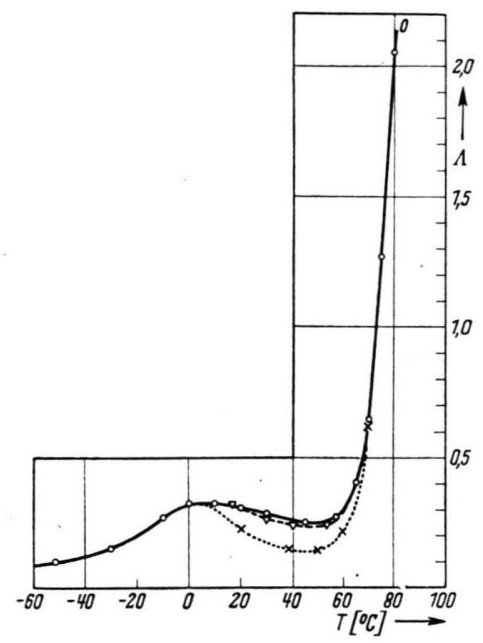

Abb. 8. Das log. Dekrement im System PMA $+3 \%$ Butylacetat. $-\Lambda_{\text {exp. }},----\Lambda_{\text {theor. mit }} \tau_{1}$ und $\tau_{2}{ }^{\prime}$ berechnet, ....... Gleichgewichtskurve mit $\tau_{1}$ und $\tau_{2}$ berechnet.

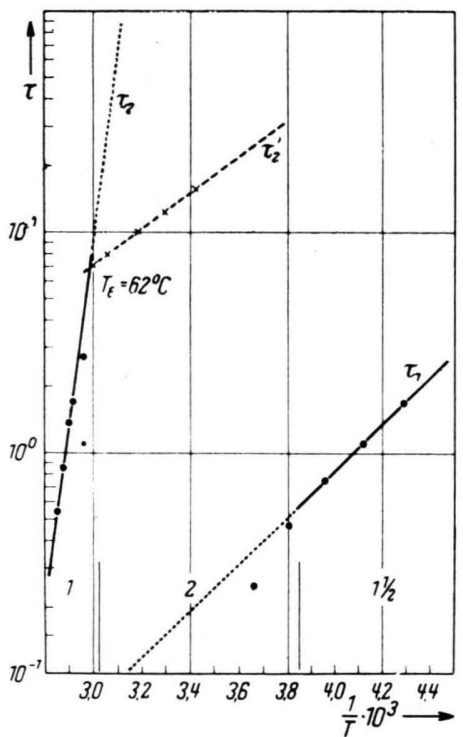

Abb. 9. Die Relaxationszeit $\tau$ im System PMA $+3 \%$ Butylacetat. 1: 1-faches, 2: 2-faches, 1 1/2:1 1/2-faches Maxwellsches Modell.

${ }^{17}$ L.V.Holroyd, R. Codrington, B. A. Mrowca u. E. Guth, J. Appl. Phys. 22, 696 [1951].

${ }^{18}$ H. A. Robinson, R. Ruggy u. E. Slantz, J. Appl. Phys. 15, 343 [1944]. 
ven an Polystyrol ausgewertet und ebenfalls die Einfriertemperatur als Knickpunkt der $\log \tau-1 / T$. Kurve gefunden ${ }^{5}$.

\section{Weichgemachte Polymerisate}

BeiWeichmacherzusatz rücken die beiden Dämpfungsmaxima mehr und mehr zusammen. Man kann dann nicht mehr aus der Höhe des kleinen Maximums das Verhältnis $D_{1} / D_{2}$ entnehmen, welches ja als Parameter in den Gln. $\left(9 \mathrm{~b}^{*}\right)$ und $\left(9 \mathrm{c}^{*}\right)$ steckt.

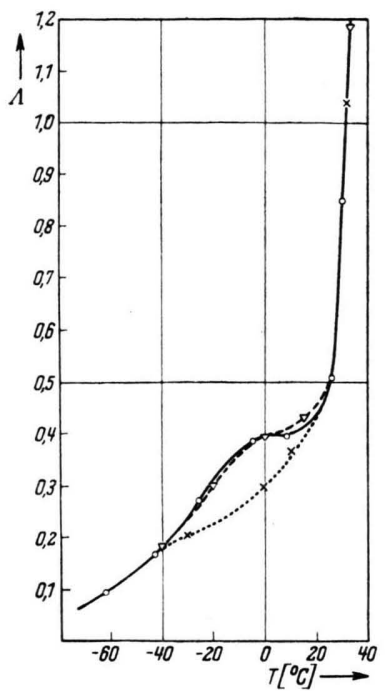

Abb. 10. Das log. Dekrement im System PMA + 15\% Butylacetat. $-\Lambda_{\text {exp. }},---\Lambda_{\text {theor. mit }} \tau_{1}$ und $\tau_{2}{ }^{\prime}$ berechnet, ....... Gleichgewichtskurve mit $\tau_{1}$ und $\tau_{2}$ berechnet.

Für eine 15 Gew.-\% Butylacetat enthaltende Probe soll die theoretische Auswertung ebenfalls mitgeteilt werden, die in der gleichen Weise durchgeführt wurde wie beim reinen PMA, mit einer einzigen zusätzlichen Annahme: Da sich die Schwingungsdauer bei sehr tiefen Temperaturen, $\vartheta_{0}$, bei gleichen Probekörpern und Trägheitsmomenten durch Zusatz von Weichmacher nicht ändert - zumindest im hier angewandten Konzentrationsbereich - ist auch wegen Gl. (7) $D_{\text {ges. }}=D_{1}+D_{2}$ konstant. Entscheidend für die Aufteilung von $D_{\text {ges. }}$ auf $D_{1}$ und $D_{2}$ ist die Höhe des kleinen Dämpfungsmaximums, die aber nicht mehr direkt zugänglich ist. Es wurde deshalb angenommen, daß sich - in Übereinstimmung mit dem Experiment - das sekundäre Dispersionsgebiet bei Weichmacherzusatz zu tieferen Temperaturen verschiebt, die zu diesem Bindungsmechanismus gehörende maximale Dämpfung jedoch vom
Weichmacherzusatz unbeeinflußt konstant bleibt. Das bedeutet, daß, nachdem $D_{\text {ges. }}=$ const gefunden wurde, auch $D_{1} / D_{2}=$ const angenommen wird. Diese Annahme stellt natürlich nur eine erste Näherung dar. Da für das sekundäre Dispersionsgebiet der PMA und nicht der Weichmacher verantwortlich ist, muß die Höhe dieses kleinen Dämpfungsmaximums und somit auch das Verhältnis $D_{1} / D_{2}$ bei Weichmacherzusatz abnehmen. Es stellt sich aber heraus, daß obige Annahme in dem zugrunde gelegten Konzentrationsbereich hinreichend genau die Beobachtungen beschreibt, wie sich gleich zeigen wird.

Die Auswertung geht wieder so vor sich, daß sich die Dämpfungskurve bei hohen bzw. tiefen Temperaturen durch ein 1-faches bzw. 1 1/2-faches Maxwellsches Modell, im dazwischen liegenden Überlagerungsgebiet jedoch nur durch ein 2 -faches Modell beschreiben läßt. Die Ergebnisse sind in den Abb. 10 und 11 dargestellt. Die Annahme konstanter Temperaturkoeffizienten für $\tau_{1}$ und $\tau_{2}$ (geradlinig extrapoliert) führt wieder zu einer erheblich

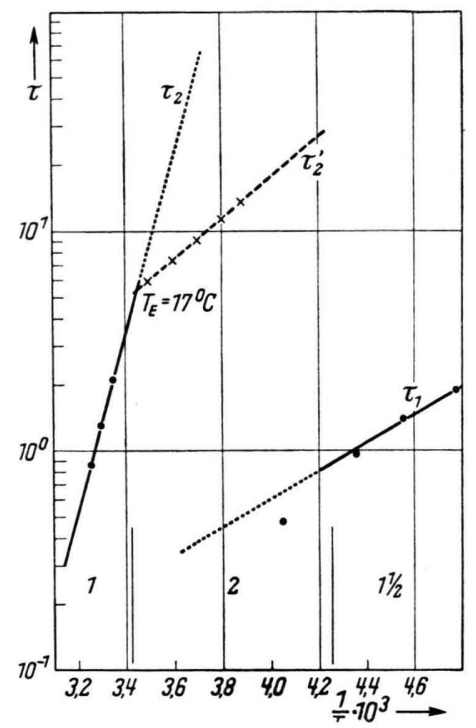

Abb. 11. Die Relaxationszeit $\tau$ im System PMA $+15 \%$ Butylacetat. 1: 1-faches, 2: 2-faches, 1 1/2: 1 1/2faches Maxwellsches Modell.

unterhalb der experimentellen liegenden theoretischen Dämpfungskurve (Abb. 10, punktiert). Diejenigen $\tau_{2}$-Werte, die beide Kurven zur Deckung bringen, liegen in Abb. 11 exakt auf einer Geraden, die die Gerade für $\tau_{2}$ bei der Einfriertemperatur $T_{\mathrm{E}}=17^{\circ} \mathrm{C}$ schneidet. 
Nimmt man eine lineare Erniedrigung der Einfriertemperatur durch Weichmacherzusatz an, so ergibt sich in unserem Fall eine mittlere Erniedrigung von $3,8^{0} /$ Gew.- $\%$ Butylacetat, während früher ${ }^{19}$ aus dem Knick der Kurve des Brechungsindex $4,8^{\circ}$ gefunden wurde. (Der Index 1 bezieht sich auf das sekundäre Dispersionsgebiet, 2 auf das Hauptmaximum. Der Index ' gehört zum eingefrorenen Glaszustand unterhalb $T_{\mathrm{E}}$.)

Berechnet man unter Zugrundelegung des einfachen Maxwellschen Modells aus den Geraden im $\ln \tau_{2}-1 / T$-Diagramm nach Gl. (13) die Aktivierungsenergie $A_{2}$ der Gleichgewichtsschmelze für die verschiedenen Konzentrationen, so sieht man, daß die Aktivierungsenergie $A_{2}$ mit zunehmendem Weichmachergehalt rasch abfällt (Tab. 2) und bei 23 Gew.- \% Lösungsmittelgehalt mit $6 \mathrm{kcal} / \mathrm{Mol}$ nur noch den etwa 3 -fachen Wert der Aktivierungsenergie des reinen Lösungsmittels besitzt (ca. 2 kcal/ Mol, aus der Temperaturabhängigkeit der Viskosität gemessen).

\begin{tabular}{|c|c|c|c|c|c|c|}
\hline \multirow{2}{*}{$\begin{array}{c}\begin{array}{c}\text { Tor- } \\
\text { sions- } \\
\text { moduli }\end{array} \\
{[\text { Gew.- } \%]}\end{array}$} & \multicolumn{6}{|c|}{$\begin{array}{l}D_{\text {ges. }}=2,28 \cdot 10^{5} \text { dyn } \mathrm{cm} ; \quad G_{\text {ges. }}=1,66 \cdot 10^{10} \text { dyn } \mathrm{cm}^{-2} ; \\
D_{1}=0,38 \cdot 10^{5} \text { dyn } \mathrm{cm} ; \quad G_{1}=0,28 \cdot 10^{10} \mathrm{dyn}^{-2} ; \\
D_{2}=1,90 \cdot 10^{5} \text { dyn } \mathrm{cm} ; \quad G_{2}=1,38 \cdot 10^{10} \mathrm{dyn}^{-2}\end{array}$} \\
\hline & $\begin{array}{c}A_{1} \\
{[\mathrm{kcal} / \mathrm{Mol}]}\end{array}$ & $\begin{array}{c}A_{2} \\
\mathrm{kcal} / \mathrm{Mol}]\end{array}$ & $\begin{array}{c}A_{2}{ }^{\prime} \\
{[\mathrm{kcal} / \mathrm{Mol}]}\end{array}$ & $\begin{array}{c}\tau_{10} \\
{[\mathrm{sec}]}\end{array}$ & $\begin{array}{c}\tau_{20} \\
{[\mathrm{sec}]}\end{array}$ & $\begin{array}{c}\tau_{20}{ }^{\prime} \\
{[\mathrm{sec}]}\end{array}$ \\
\hline \multicolumn{7}{|c|}{ Butylacetat } \\
\hline $\begin{array}{l}3 \\
5,2 \\
10,7 \\
13,4 \\
15 \\
23\end{array}$ & $\begin{array}{l}3,84 \\
3,66\end{array}$ & $\begin{array}{l}36,2 \\
34 \\
20,3 \\
21,4 \\
18,3 \\
6,1\end{array}$ & 3,38 & $1,6 \cdot 10^{-3}$ & $\begin{array}{l}10^{-22,7} \\
10^{-21,7} \\
10^{-13,6} \\
10^{-14,9} \\
10^{-13} \\
10^{-4,5}\end{array}$ & $\begin{array}{l}3,7 \cdot 10^{-2} \\
2,6 \cdot 10^{-3}\end{array}$ \\
\hline \multicolumn{7}{|c|}{ Chloroform } \\
\hline $\begin{array}{r}4 \\
10 \\
12 \\
14 \\
16 \\
22\end{array}$ & $\begin{array}{l}3,45 \\
3,14\end{array}$ & $\begin{array}{l}31 \\
25,4 \\
20,3 \\
19,1 \\
15,7 \\
8,15\end{array}$ & & $\frac{10^{-2,25}}{10^{-2,14}}$ & $\begin{array}{l}10^{-19,6} \\
10^{-16,4} \\
10^{-13,3} \\
10^{-12,8} \\
10^{-10,9} \\
10^{-5,9}\end{array}$ & \\
\hline
\end{tabular}

Tab. 2. Zusammenfassung der Ergebnisse für die Systeme PMA-Bu tylacetat und PMA-Chloroform $\left(\vartheta_{0}=2 \mathrm{sec} ; \Theta=2,28 \cdot 10^{4} \mathrm{gr} \mathrm{cm}^{2}\right.$. $\left.D_{1} / D_{2}=0,198\right)$.

Bestimmt man ferner für die verschiedenen Weichmacherkonzentrationen die Relaxationszeit bei sehr hohen Temperaturen $\tau_{20}$ (Tab. 2), so ergibt sich ein linearer Zusammenhang zwischen $\log \left(\mathbf{1} / \tau_{20}\right)$ und der Aktivierungsenergie $A_{2}$ über rund $20 \mathrm{Zeh}$ nerpotenzen (Abb. 12).

$$
\log \left(1 / \tau_{20}\right)=0,57 A_{2}+2 .
$$

${ }^{19}$ E. Jenckel u. R. Heusch, Kolloid-Z. 130, 89 [1953].
Eine Verringerung von $\tau_{2}$ durch Verringerung von $A_{2}$ wird also teilweise wieder dadurch ausgeglichen, daß gleichzeitig $\tau_{20}$ größer wird. Eine Beeinflussung in der gleichen Richtung fordert auch die Theorie von Eyring und Tobolski ${ }^{20}$.

Es ist interessant, daß ein solcher Zusammenhang ganz allgemein bei der heterogenen Katalyse zwischen Reaktionsentropie und Aktivierungsenergie gefunden wird ${ }^{21}$.

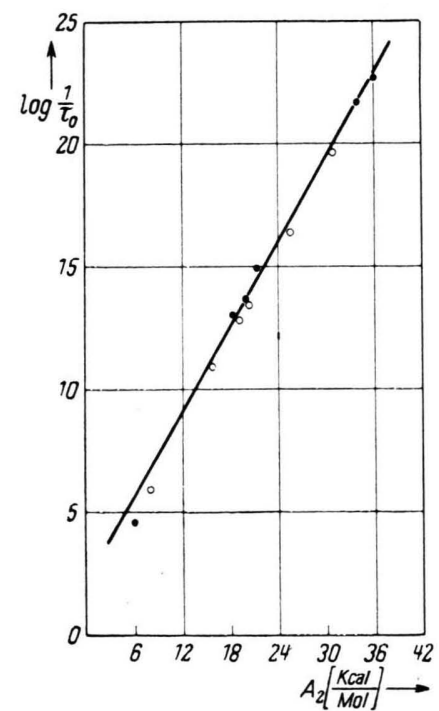

Abb. 12. Relaxationszeit $\tau$ und Aktivierungsenergie $A_{2}$. Erniedrigung von $A_{2}$ durch Butylacetat $\bullet$ und Chloroform $O$ als Weichmacher.

III. Die Einfriertemperatur und die Temperatur des Dämpfungsmaximums

Verfolgt man bei der Abkühlung eines geschmolzenen Stoffes, bei dem Kristallisation unterbleibt und schließlich glasige Erstarrung eintritt, das Volumen, so beobachtet man einen großen Temperaturkoeffizienten bei hohen Temperaturen, jedoch einen sehr viel kleineren unterhalb einer gewissen Temperatur, der sogenannten Einfriertemperatur. Die Kurve des gegen die Temperatur aufgetragenen Volumens wird also durch zwei sich schneidende Geraden dargestellt. Man kann zeigen, daß oberhalb der Einfriertemperatur die beobachteten Volumina Gleichgewichtswerte sind, während unterhalb der Einfriertemperatur der Stoff das Bestreben hat, sein Volumen zu vermindern, bis er schließlich die Werte der Gleichgewichtsgeraden erreicht, ein Ver-

${ }^{20}$ W. J. Lyons, J. Appl. Phys. 23, 287 [1952].

21 E. Cremer, Z. Elektrochem. 53, 269 [1949]. 
such, der praktisch allerdings nur wenige Grade unterhalb der Einfriertemperatur durchzuführen ist. Die gleichen Beobachtungen werden am Wärmeinhalt gemacht, dessen Kurve bei der Einfriertemperatur einen Knickpunkt aufweist, bzw. an der spezifischen Wärme, welche bei der Einfriertemperatur fast sprunghaft abnimmt. Die Einfriertemperatur ist also dadurch ausgezeichnet, daß sich bei ihr die Temperaturkoeffizienten gewisser Eigenschaften fast sprunghaft ändern, derart, daß unterhalb dieser Temperatur kleine Temperaturkoeffizienten, oberhalb große Temperaturkoeffizienten beobachtet werden.

Über das molekulare Geschehen, insbesondere auch in hochpolymeren Lösungen (weich gemachte Polymerisate), vgl. ${ }^{19}$.

Unterwirft man dagegen den - glasigen, flüssigen oder kristallinen - Stoff bei verschiedenen Temperaturen mechanischen oder elektrischen Schwingungen, so beobachtet man bei freien Schwingungen ein Maximum der Dämpfung bei gewissen Temperaturen, wie z. B. oben gezeigt wurde, und das gleiche gilt für die Energieverluste bei erzwungenen Schwingungen. Wir wollen hier nur die Dämpfung der freien Torsionsschwingungen näher betrachten, jedoch lassen sich diese Gedankengänge sinngemä $B$ ohne weiteres auf die erzwungenen Schwingungen übertragen.

Berechnet man die Dämpfungskurve mit Hilfe des $1 \frac{1}{2}$-fachen Maxwell-Modells, so zeigt sich in aller Schärfe, daß das Maximum durch die Korrespondenz von Schwingungsdauer $\vartheta$ und Relaxationszeit $\tau$ bedingt ist*, während der Begriff der Einfriertemperatur in dieser Rechnung überhaupt nicht vorkommt.

Unsere Messungen zeigen nun deutlich nebeneinander einerseits das Maximum von $\Lambda$ bei einer bestimmten Temperatur, d. h. bei einer bestimmten Relaxationszeit, bei gegebener Schwingungsdauer und andererseits die sprunghafte Abnahme des Temperaturkoeffizienten einer Eigenschaft des Stoffes, in diesem Falle der Relaxationszeit $\tau_{2}$ unterhalb der Einfriertemperatur. Die Existenz einer Einfriertemperatur wird noch dadurch unterstrichen, daß

\footnotetext{
* Eben wegen dieses engen Zusammenhanges zwischen Schwingungsdauer und Relaxationszeit sollte sich das Maximum auf den Kurven recht stark mit verschieben. Das ist jedoch nur geringfügig der Fall. Immerhin haben Schmieder und Wolf ${ }^{9}$ und Stavermann und Mitarbb. ${ }^{22}$ ein wenn auch recht breites Maximum der Dämpfung gegen die Schwin-
}

man bei langsamem Abkühlen oder Tempern Dämpfungskurven mit tieferem Minimum erhält. Um diesen Befund zu verstehen, muß man einen größeren $\tau_{2}$-Wert annehmen. D. h. aber: Der Wert $\tau_{2}$ hat sich in Richtung auf den bei geradliniger Extrapolation aus der Schmelze oberhalb der Einfriertemperatur zu erwartenden Wert bewegt. Dieses Verhalten ist typisch für den Einfriervorgang.

Man darf also Temperatur des Dämpfungsmaximums und Einfriertemperatur nicht einander gleichsetzen, was durchweg geschieht und auch von uns selbst vor wenigen Jahren noch so dargestellt wurde. Das Material ist nicht eingefroren, weil es bei hinreichend kurzer Schwingungsdauer inneren Bewegungen und Verschiebungen zu folgen keine Zeit hat; diesem Umstand wird bereits durch die oben verwendete Formel von $\Lambda=f\left(\tau, D_{1} / D_{2}, \vartheta_{0}\right)$ Rechnung getragen; er führt zu einer verminderten Dämpfung. Das Material friert glasig ein, während es von einer Temperatur auf die andere abgekühlt wird. Erst dabei entsteht die oben skizzierte charakteristische Struktur des Glases.

\section{Experimentelles}

Der durch Polymerisation mit Benzoylperoxyd als Katalysator gewonnene PMA wurde durch fraktionierte Fällung mit destilliertem Wasser und Lösung in Aceton sorgfältig in 4 Fraktionen unterteilt, deren Molekulargewichte viskosimetrisch in Chloroform-Lösung von 0,5 bis $3 \mathrm{~g} / l$ bestimmt und nach den Formeln

$$
Z_{\eta}=\frac{\eta_{\text {spez. }}}{c}\left(\frac{1}{1+K_{\eta} \cdot \eta_{\text {spez. }}}\right) \quad \text { mit } K_{\eta}=0,29
$$

und $Z_{\eta}=K M^{\alpha}$ mit $\alpha=0,81$ und $K=5,1 \cdot 10^{-6}$ berechnet wurde; Mittelwerte aus vielen Messungen ergaben

\begin{tabular}{|c|c|c|c|c|}
\hline Fraktion & I & II & III & IV \\
\hline$M$ & 600000 & 300000 & 100000 & 34000 \\
\hline
\end{tabular}

mit einem Fehler von $\pm 2 \%$.

Zur Herstellung der Probekörper wurden die Fraktionen in den untersuchten Lösungsmitteln gelöst und durch Abdampfen des Lösungsmittels auf einer Quecksilberoberfläche Folien gewonnen, die in Streifen geschnitten wurden. Die Dimensionen der Probekörper betrugen im Durchschnitt: $l=15 \mathrm{~cm}, b=0,8 \mathrm{~cm}, d=$ $0,1 \mathrm{~cm}$. Ohne Zerstörung der Folien war es nicht mög-

gungsdauer überzeugend herleiten können. Dieses rührt offenbar nur von einer sehr breiten Verteilung der Relaxationszeiten her. Es ist jedoch bislang nicht möglich gewesen, mit unserer Vorstellung $\Lambda=f(\vartheta)$ bei $\tau_{i}=$ const, d. h. bei $T=$ const zu berechnen.

22 A. J. Stavermann, J. Heyboer u. P. Dokking, Reprints Oxford 1953, S. 200. 
lich, einen Lösungsmittelrest von ca. 3 Gew.- \% aus ihnen zu entfernen. Der Lösungsmittelrest wurde nachgewiesen durch scharfes Tempern einer sehr dünnen Folie bei $190^{\circ}$ unter einem Druck von etwa $10^{-1}$ Torr, wobei nach Grassie ${ }^{23}$ noch kein Abbau stattfindet. Er mußte bei der Auswertung in Rechnung gestellt werden. Die Fraktion mit den kürzesten Ketten $(P=$ 340 ) konnte nicht verwandt werden, da sie so spröde war, daß sie bei der geringsten Beanspruchung zersprang.

\section{MeBapparatur}

Zur Messung diente ein Torsionspendel, wie es in Abb. 13 beschrieben ist. Der Probekörper war unten fest eingespannt und oben an einem Faden von möglichst geringer Torsionssteifigkeit so über 2 Rollen frei aufgehängt, daß er sich mit der beweglichen Hülse $H$ in vertikaler Richtung ohne Änderung der Längsspannung verschieben ließ. Die Messungen wurden ohne Aufbringen einer Längsspannung ausgeführt. Geringfügige Änderungen in $b$ und $d$ sowie eine Variation von $l$ zwischen $20 \mathrm{~cm}$ und $4 \mathrm{~cm}$ hatten keinen Einflu $\beta$ auf die Meßergebnisse.

Bei hohen Temperaturen wurde das Torsionspendel in einen Heizofen eingefahren, bei tiefen Temperaturen mit einem Schutzrohr versehen und in ein Kältebad getaucht. Die Temperaturkonstanz betrug mindestens $\pm 0,5^{\circ} \mathrm{C}$. Gemessen wurde durchweg von hohen $\mathrm{zu}$ tiefen Temperaturen mit einer Abkühlungsgeschwindigkeit von $0,5^{0} / \mathrm{min}$. Die Messung wurde erst ausgeführt, nachdem die Probe 15 min bei der betreffenden Temperatur gewesen war.

${ }^{23}$ N. Grassie, J. Polymer Sci. 6, 643 [1951].
Das log. Dekrement wurde bestimmt aus dem Verhältnis zweier um eine Schwingungsperiode auseinanderliegender Amplituden. Die Schwingungsdauer wurde mit einer Stoppuhr zwischen zwei in gleicher Richtung verlaufenden Nulldurchgängen des Lichtzeigers gemessen. $\Lambda$ und $\vartheta$ waren über den Verlauf einer gesamten Schwingung konstant. Die Schwingungsdauer $\vartheta$ konnte durch Veränderung des Trägheitsmomentes $\eta$ mittels Verschieben der Gewichte g auf dem Querbalken T beeinflußt werden.

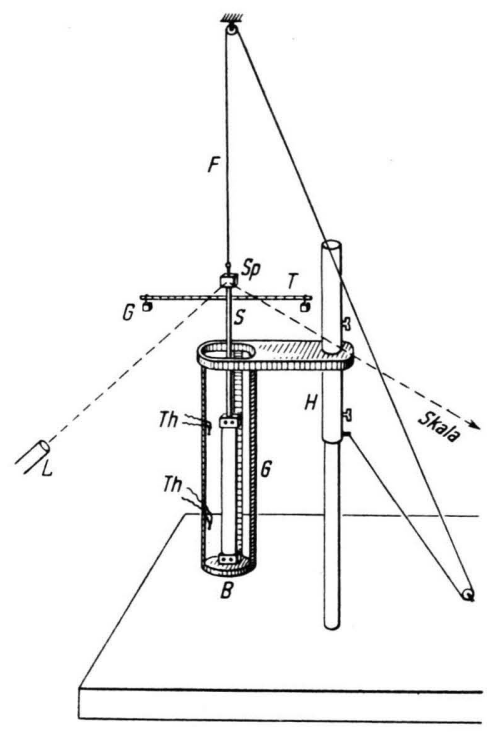

Abb. 13. Torsionspendel.

\title{
Über ein eindimensionales Modell halbleitender Verbindungen vom Typus $\mathrm{A}^{\mathrm{II}} \mathrm{B}^{\mathrm{V}}$
}

\author{
Von B. Seraphin \\ Aus dem Forschungslaboratorium der Siemens-Schuckertwerke A.-G., Erlangen \\ (Z. Naturforschg. 9a, 450-456 [1954]; eingegangen am 18. März 1954)
}

Im eindimensionalen Potentialmodell wird das Verhalten der Bindungsfestigkeit, der Breite der verbotenen Zone und der Beweglichkeit von Löchern und Elektronen betrachtet, wenn man vom Fall gleicher Potentialmulden zu einer Anordnung übergeht, in der jeweils eine tiefere und eine flachere Mulde aufeinander folgen. Es wird qualitative Übereinstimmung mit den Beobachtungen innerhalb einer isoelektronischen Reihe erzielt, insbesondere bezüglich des Vergleichs der AIIIBV-Verbindungen mit dem isoelektronischen Element.

$\mathrm{D}^{\mathrm{i}}$ e Halbleitereigenschaften der Verbindungen vom Typus $\mathrm{A}^{\mathrm{III}} \mathrm{B}^{\mathrm{V}}$ weichen von denen der vierten Gruppe in charakteristischer Weise ab. Die wesentlichsten dieser systematischen Abweichungen von den isoelektronischen Elementen der vierten

${ }^{1}$ H. Welker, Z. Naturforschg. 7a, 744 [1952], Sa, 248 [1953].
Hauptgruppe ( $\mathrm{Si}, \mathrm{Ge}, \alpha$-Sn) lassen sich in folgenden vier Punkten zusammenfassen ${ }^{1,2}$ :

1. Die Breite der verbotenen Energiezone zwischen Valenz- und Leitungsband nimmt zu.

2. Die Elektronenbeweglichkeit steigt an. 